\title{
Ultrasound enhanced synthetic platelet therapy for augmented wound repair
}

Seema Nandi ${ }^{1,2}$; Kaustav Mohanty ${ }^{3}$; Kimberly Nellenbach ${ }^{1,2}$, Mary Erb $^{1}$; Marie Muller ${ }^{1,3}$; Ashley C. Brown ${ }^{1,2^{*}}$

${ }^{1}$ Joint Department of Biomedical Engineering, North Carolina State University and The University of North Carolina at Chapel Hill, Raleigh, NC

${ }^{2}$ Comparative Medicine Institute, North Carolina State University

${ }^{3}$ Department of Mechanical and Aerospace Engineering, North Carolina State University

\section{"Correspondence:}

Ashley C. Brown, $\mathrm{PhD}$

Joint Department of Biomedical Engineering

University of North Carolina at Chapel Hill/North Carolina State University

1001 William Moore Dr.

Raleigh, NC, 27607

aecarso2@ncsu.edu

Contents: Five Supplemental Figures 

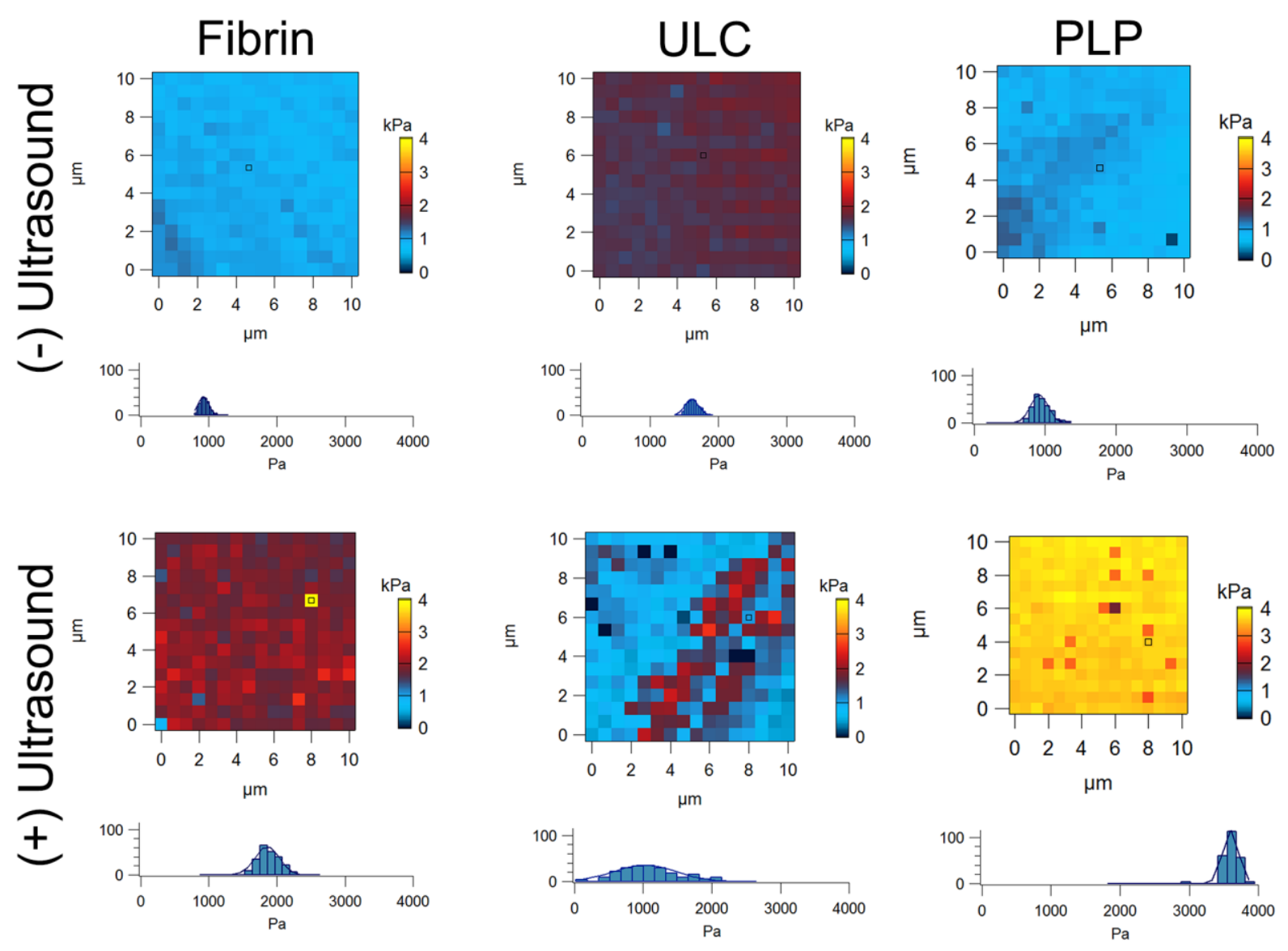

Figure S1: Representative force maps of fibrin clots in the presence or absence of ultrasound.

Clots containing PLPs and exposed to ultrasound show higher stiffness values when measured via atomic force microscopy nanoindentation than all other clot groups 2 hours after initial clot formation. $\mathrm{n}=3$ clots per group with 3 random locations imaged per clot. 

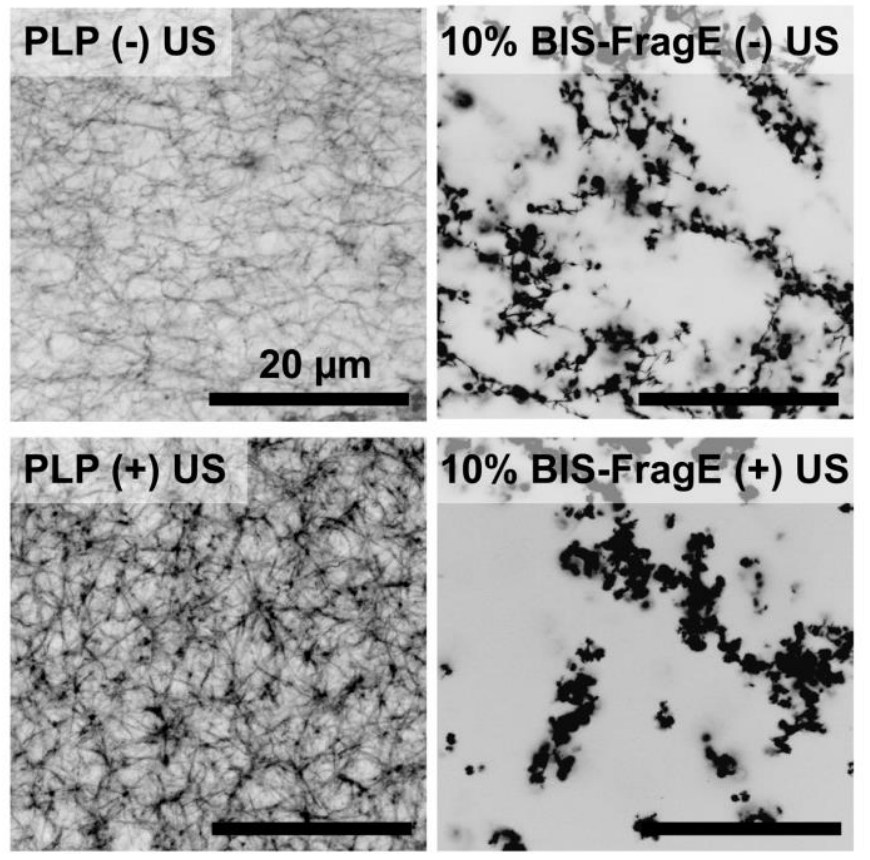
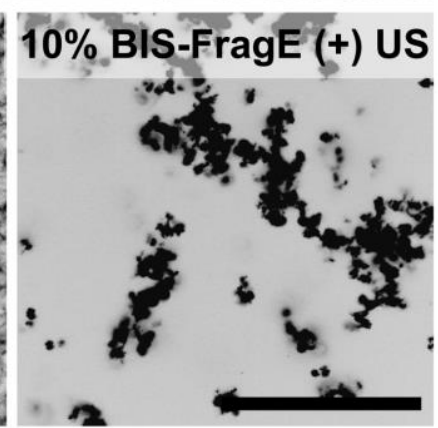

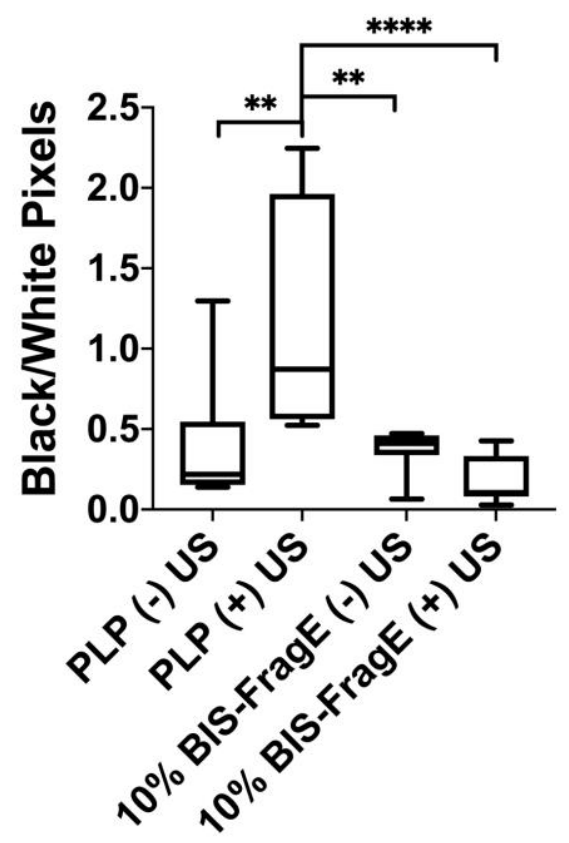

Figure S2: Comparison of PLP + US and hard (10\% BIS) fibrin-targeting particle effects on clot density. PLPs + US result in increased clot network density over both PLPs alone and fibrintargeting hard particles with or without the addition of ultrasound stimulation. $\mathrm{n}=3$ clots/group, 3 random z-stacks imaged/clot. $* * \mathrm{p}<0.01 ; * * * \mathrm{p}<0.0001$. 

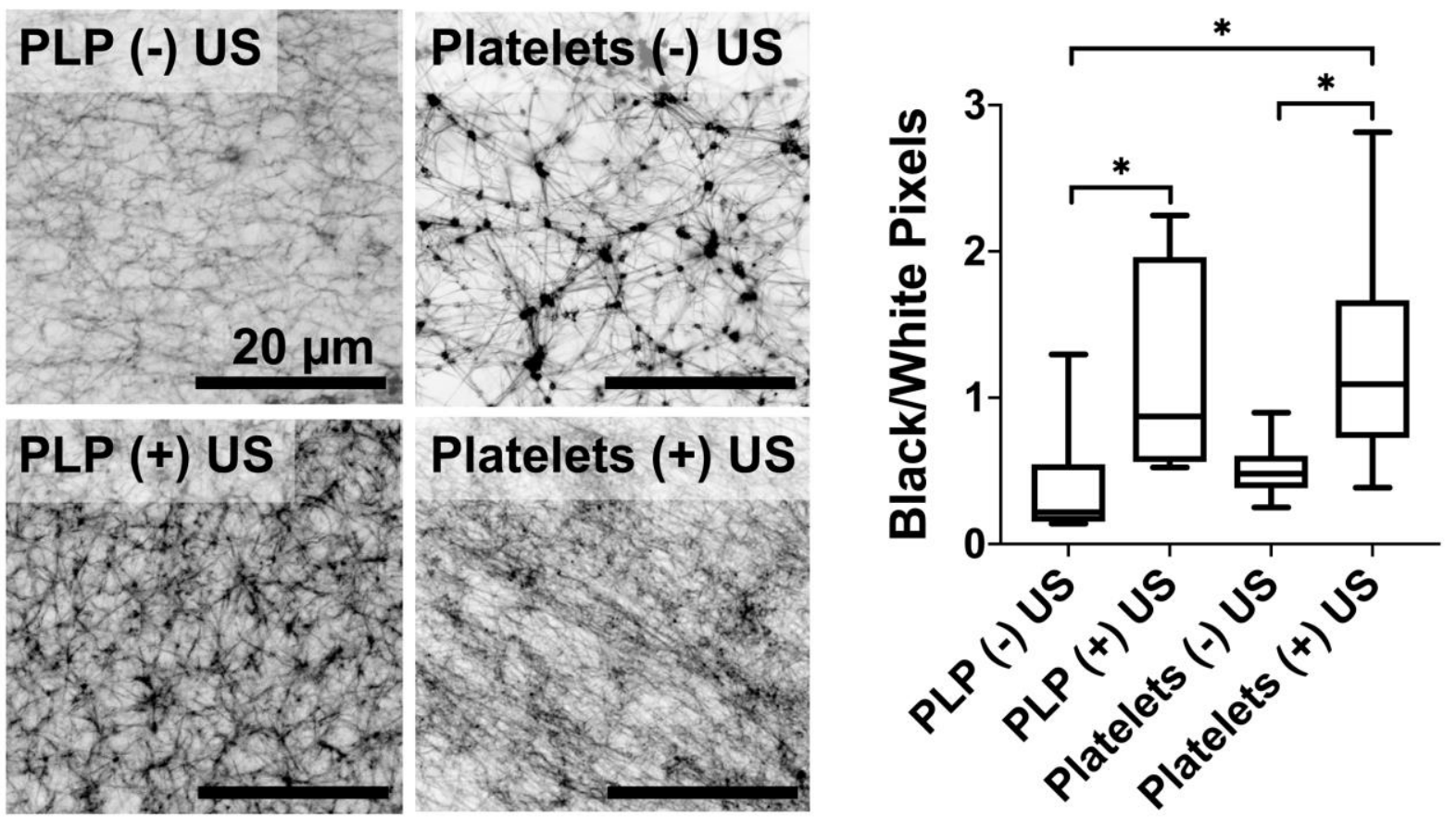

Figure S3: Comparison of PLP + US and native platelet effects on clot density. PLPs + US

result in increased clot network density over both PLPs alone and platelets alone, while resulting in similar clot network densities in comparison to native platelets + US. $\mathrm{n}=3$ clots/group, 3 random z-stacks imaged/clot. ${ }^{*} \mathrm{p}<0.05$. 


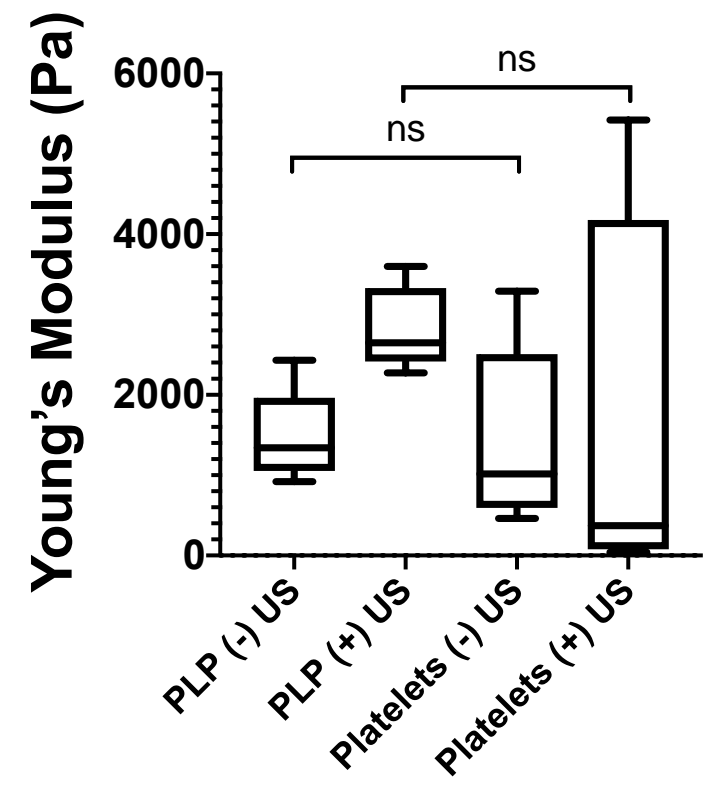

Figure S4: Comparison of PLP + US and native platelet effects on clot stiffness. No significant differences were observed between clot stiffness in clots containing PLPs vs. clots containing native platelets. $\mathrm{n}=3$ clots/group, 3 random locations imaged/clot, 256 force curves obtained/image. ns: $\mathrm{p}>0.05$. 


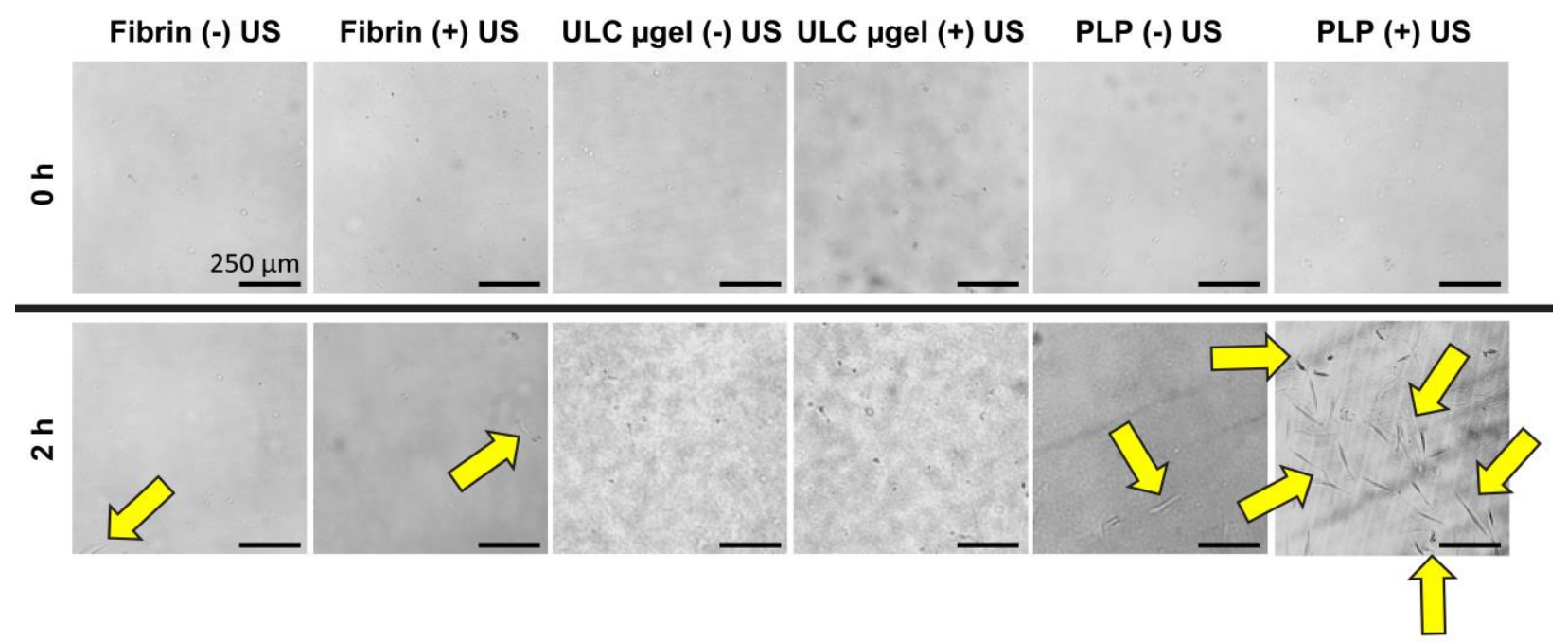

Figure S5: Representative images of "wounded" area in fibroblast migration assay. At $t=0$ hours after clot application, no cells were observed in the wound area. At $\mathrm{t}=2$ hours after clot application, groups treated with combinatorial PLPs + US displayed greater fibroblast presence within the provisional clot matrix relative to control groups treated with fibrin-only clots or fibrin clots containing non-fibrin targeting ULC microgels, which contained very few cells (marked with arrows) within the applied provisional clot matrices. $\mathrm{n}=6$ per condition. 